AUC for 30 day mortality for the acute score was 0.84 (95\% CI $0.80-0.88)$.

The strongest predictors of 1 year mortality were age $>80$ years HR 1.25 (95\%CI 1.00-1.64)-1 point, neoplastic disease 1.91 (95\%CI 1.37-2.65)-1 point, MRC dyspnoea grade 4 or 5 (3.82 95\%CI 2.61-5.58)- 2 points, $\mathrm{FEV}_{1}<30 \%$ predicted 2.61 (95\%CI 1.99-3.43)-1 point, long term oxygen therapy 1.60 95\%CI 1.11-2.29)-1 point and a history of myocardial infarction or heart failure $2.7695 \% \mathrm{CI} 1.70-4.47)-1$ point. The AUC for 1 year mortality of the chronic score was 0.76 (95\% CI $0.73-0.80)$.

The resulting 4 stage model identifies different outcomes within each subgroup (See Figure 1). Furthermore the 4 stage model predicted 30-day mortality AUC 0.76 (95\%CI $0.72-$ $0.79)$, 1 year mortality $0.72(95 \% \mathrm{CI} 0.70-0.74)$ and readmissions 0.74 (95\%CI 0.72-0.76) better than GOLD 2011 criteria. Conclusion A multidimensional prognostic index can predict both short and long term outcomes after COPD exacerbations, and divides patients into clinically useful subgroups based on exacerbation severity and chronic health status.

\section{P137 INTERNAL VALIDATION OF THE DECAF SCORE AS A PREDICTOR OF INPATIENT MORTALITY IN ACUTE EXACERBATIONS OF COPD}

${ }^{1} \mathrm{C}$ Echevarria, ${ }^{1} \mathrm{~J}$ Steer, ${ }^{2} \mathrm{G}$ I Gibson, 'S C Bourke; 'North Tyneside General Hospital, North Shields, United Kingdom; ${ }^{2}$ Newcastle University, Newcastle Upon Tyne, United Kingdom

\subsection{6/thoraxjn-2013-204457.287}

Introduction In patients presenting with an acute exacerbation of COPD (AECOPD), accurate prediction of in-hospital mortality may help inform the most appropriate place and level of care. The DECAF score was developed for this purpose and designed to be simple to apply at the bedside using variables that are routinely collected on admission: Dyspnoea, Eosinopenia, Consolidation, Acidaemia and atrial Fibrillation. Whilst the performance of the tool within the derivation cohort was strong,[1] before recommending use in clinical practice further validation is required.

Methods Both external and internal validation of the DECAF score are currently in progress; for each, a cohort of 840 patients consecutively admitted with AECOPD is being recruited. To optimise data capture, patients are identified by screening admissions units and coding records. Indices that comprise the DECAF score and other independent predictors of mortality and readmission are collected. Dyspnoea is assessed using the extended MRC dyspnoea score.[1] Inclusion criteria are: primary diagnosis of AECOPD, age 35 or older, 10 pack years or more smoking history, and obstructive spirometry. Exclusion criteria are: other illness likely to limit survival to less than one year (principally metastatic malignancy) and previous inclusion in the validation study. We present an analysis of the performance of DECAF in the first 623 patients recruited to the internal validation cohort. Results

\section{Abstract P137 Table 1. \\ DECAF AUROC $=0.82(95 \% 0.76$ to 0.87$)$ \\ Derivation DECAF AUROC $=0.86(95 \% \mathrm{Cl} 0.82$ to 0.89$)$ \\ $\mathrm{p}$ value using Fisher's exact test Internal validation DECAF AUROC $=0.82(95 \% 0.76$ to \\ 0.87) Derivation DECAF AUROC $=0.86$ (95\% Cl 0.82 to 0.89$)$}

Discussion As in the derivation study, DECAF is a good predictor of inpatient mortality (AUROC $=0.82$ ), with a stepwise increase in mortality with DECAF score. The DECAF score accurately identifies low risk (DECAF score 0-1) and high risk patients (3 or greater) admitted with an exacerbation of COPD, potentially helping select patients for early supported discharge schemes, or for intensified medical treatment or early palliation.

\section{REFERENCES}

1. Steer, J., J. Gibson, and S. C. Bourke, The DECAF Score: predicting hospital mortality in exacerbations of chronic obstructive pulmonary disease. Thorax, 2012. 67(11): p. $970-6$.

\section{P138 MODERATE-INTENSITY ACTIVITY IS ASSOCIATED WITH REDUCED CARDIOVASCULAR RISK FACTORS IN COPD}

${ }^{1}$ AM Albarrati, ${ }^{1} \mathrm{NS}$ Gale, ${ }^{2} \mathrm{~S}$ Enright, ${ }^{1} \mathrm{IC}$ Munnery, ${ }^{1} \mathrm{MM}$ Munnery, ${ }^{1} J \mathrm{R}$ Cockcroft, ${ }^{1} \mathrm{DJ}$ Shale; ${ }^{1}$ Cardiorespiratory Medicine, Cardiff University, Cardiff, United Kingdom; ${ }^{2}$ Physiotherapy Department, Cardiff University, Cardiff, United Kingdom

\subsection{6/thoraxjnl-2013-204457.288}

Background Steps measurement is a common measure of daily physical activity, but cannot define the intensity of physical activity. Moderate intensity activity is recommended type to maintain a basic level of health (1), but in patients with COPD, the effect of moderate intensity activity on systemic manifestations has not been studied. We hypothesised that patients with higher moderate activity time would have lower frailty, inflammation and cardiovascular risk.

Methods As part of a longitudinal study in COPD (ARCADE), daily physical activity was recorded over seven days using a multisensor armband (SenseWear Pro armband) in 75 stable patients with COPD. Spirometry, body composition, aortic stiffness, comprehensive geriatric assessment, C-reactive protein (CRP) and fibrinogen were also determined. Moderate-intensity activity was determined by the monitor for activity between 3 to 6 METs.

Results Patients (42 males) mean (SD) age was 66 (7) years, BMI $27.5(5.2) \mathrm{Kg} / \mathrm{m}^{2}, \mathrm{FEV}_{1}$ predicted $55(17) \%$ and moderate activity time 1.46 (1.23) hours. The time spent on moderate activities was not related to age or $\mathrm{FEV}_{1}$ pred. The moderate activity time related to BMI $(r=-0.41)$, fat mass $(r=-0.38)$ and fat percentage $(r=-0.37)$, all $\mathrm{p}<0.001$, but not with fat free mass. However, none of these parameters related to the number of steps. Moderate activity time was inversely associated with aortic stiffness, $\mathrm{r}=-0.31, \mathrm{p}<0.01$, CRP, fibrinogen $(\mathrm{r}=$ $0.26 ; \mathrm{r}=-0.24$, respectively) and frailty score all $\mathrm{p}<0.05$. However, the number of steps only related to the inflammatory markers.

Conclusion The changes in frailty and cardiovascular risk factors including adipose tissue, aortic stiffness and inflammatory markers are linked to the proportion of time in moderate activities as their predominant form of activity.

\section{REFERENCES}

1. Elsawy, B and Higgins, K. (2010) Am Fam Physician 1;81(1):55-59.

\section{Mechanisms of lung injury}

\section{P139 PULMONARY SURFACTANT PROTECTS AGAINST SILVER NANOPARTICLE-INDUCED INFLAMMATION IN THE PERIPHERAL HUMAN LUNG}

S Nestorowa, TD Tetley, AJ Thorley; NHLI, Imperial College London, London, United Kingdom

10.1136/thoraxjnl-2013-204457.289 
Introduction The development and use of nanoparticles for medical applications is rapidly increasing. One of the most commonly used nanoparticles is silver, due to its antimicrobial properties. At present the use of silver nanoparticles is unregulated and there is increasing concern regarding their potential adverse health effects, particularly when inhaled. The alveolar region is the primary site of nanoparticle deposition following inhalation, thus it is important to consider the effects they will exert on the alveolar epithelium, including potential toxicity. The alveolar epithelium is covered with a surfactant layer, acting as a barrier to inhaled particulate matter and pathogens, with which nanoparticles will interact before reaching the epithelium. Thus, we hypothesise that the surfactant layer will influence the toxicity and inflammatory response of alveolar epithelial cells to inhaled AgNPs.

Methods Immortalised human alveolar type 1 epithelial (TT1) cells were exposed to increasing concentrations of $20 \mathrm{~nm}$ or $110 \mathrm{~nm}$ silver nanoparticles (AgNP) in the presence and absence of porcine surfactant (Curosurf) for 24 hours. Cell viability was measured using the MTS assay and IL-6 and IL-8 release was measured by ELISA. In addition, the effect of the antixodiant glutathione on cytokine release was assessed.

Results Exposure to $20 \mathrm{~nm}$ and $110 \mathrm{~nm}$ AgNP did not significantly affect cell viability in the presence or absence of Curosurf $(0-50 \mu \mathrm{g} / \mathrm{ml})$. However, IL-6 and IL-8 release was significantly increased $(\mathrm{P}<0.0005)$ for all AgNP at concentrations above $10 \mu \mathrm{g} / \mathrm{ml}$. Furthermore, preincubation of AgNP with Curosurf significantly inhibited this response $(\mathrm{P}<0.001)$. Pre-treatment of cells with glutathione also significantly inhibited IL-6 and IL-8 release following AgNP exposure. When glutathione and Curosurf were combined there was a further inhibition of cytokine release.

Conclusion This study demonstrates that AgNP, whilst not overtly toxic, induce an inflammatory response in human alveolar epithelial cells that is driven by oxidative stress. Furthermore, we have shown that the presence of surfactant significantly attenuates the inflammatory response suggesting a protective effect against inhaled nanoparticles. Thus, this study demonstrates the importance of studying AgNP bioreactivity in the presence of lung secretions to accurately represent the likely effects of inhalation.

\section{P140 BPIFB1/LPLUNC1 IS A NOVEL MARKER FOR THE BRONCHIOLISED EPITHELIUM IN IPF}

${ }^{1} \mathrm{CD}$ Bingle, ${ }^{1} \mathrm{~B}$ Araujo, ${ }^{2}$ WA Wallace, ${ }^{1} \mathrm{HM}$ Marriott, ${ }^{2} \mathrm{~N}$ Hirani, ${ }^{1} \mathrm{~L}$ Bingle; ${ }^{1}$ University of Sheffield, Sheffield, UK; ${ }^{2}$ University of Edinburgh, Edinburgh, UK

\subsection{6/thoraxjnl-2013-204457.290}

Idiopathic pulmonary fibrosis (IPF) is an irreversible and progressive lung disease with limited life expectancy after diagnosis. Histopathological studies of IPF lungs reveal the typical "Usual Interstitial Pneumonia” (UIP) pattern, with epithelial hyperplasia, areas of scarring with fibroblast foci and characteristic morphological abnormalities, including bronchiolization of alveolar ducts and honeycomb cysts. Although it seems likely that bronchiolar abnormalities are caused by changes in epithelial cell differentiation, specific markers of this process remain elusive. By analysis of published array data sets from IPF patients, we identified BPIFB1/LPLUNC1 as a potential candidate marker for the disease. Indeed, in the largest published study the gene was the most differentially expressed transcript. This putative innate defence protein is normally expressed in the airway submucosal glands and in a population of MUC5A/C positive goblet cells in the upper airways. Analysis of lung tissue from UIP revealed strong staining of BPIFB1/LPLUNC1 within the bronchiolized epithelium lining the honeycomb cysts as well as in the mucosubstance filling these regions. MUC5B was localized to the same cells as BPIFB1/LPLUNC1 wheras the related protein, BPIFA1/SPLUNC1, was not co-expressed. This pattern of staining was not seen in other chronic lung diseases, suggesting a degree of specificity for IPF. To shed light on a temporal association of expression of these markers with fibrosis development we studied mice exposed to the pro-fibrotic agent bleomycin (Bleo). MUC5B and LPLUNC1 were co-expressed in a population of goblet cells in the airways of mice within 3-7 days of Bleo exposure, prior to the onset of fibrosis. Continued expression was seen during the development of fibrosis between 14-21 days post treatment. In contrast, in mice treated with PBS neither protein was seen (due to mouse airways being essentially free of goblet cells). Staining was absent from the fibrotic regions and the lung parenchyma, as is the case in IPF. Our data show that the ectopic expression seen in human IPF, is mirrored by that seen in the fibrotic mouse model. Furthermore it suggests that BPIFB1/LPLUNC1 may be worthy of further study as potential marker for the disease.

\section{P141 DIFFERENTIAL EXPRESSION OF CONVENTIONAL AND INHIBITORY VEGFA ISOFORMS IN NORMAL AND FIBROTIC FIBROBLASTS-A POTENTIAL ROLE IN IPF PATHOGENESIS?}

${ }^{1} \mathrm{SL}$ Barratt, ${ }^{1} \mathrm{~T}$ Blythe, ${ }^{1} \mathrm{C}$ Jarrett, ${ }^{1} \mathrm{~K}$ Ourradi, ${ }^{2} \mathrm{~T}$ Maher, ${ }^{1} \mathrm{Gl}$ Welsh, ${ }^{3} \mathrm{DO}$ Bates, ${ }^{1} \mathrm{AB}$ Millar; ${ }^{1}$ Academic Respiratory Unit, School of Clinical Sciences, Bristol University, Bristol, UK; ${ }^{2}$ Imperial College, London, UK; ${ }^{3}$ Microvascular Research Laboratories, Bristol University, Bristol, UK

\subsection{6/thoraxjnl-2013-204457.291}

Introduction Vascular endothelial growth factor (VEGFA) has been implicated in the pathogenesis of Idiopathic Pulmonary Fibrosis (IPF). Two families of endogenous isoforms exist formed by alternative splicing of mRNA transcripts: the conventional potent angiogenic and mitogenic isoforms $\left(\mathrm{VEGF}_{\mathrm{xxx}} \mathrm{a}\right.$ family) and the $\mathrm{VEGF}_{\mathrm{xxx}} \mathrm{b}$ family that is thought to have contrasting inhibitory functions.

Hypothesis We hypothesise that differential expression of $\mathrm{VEGF}_{\mathrm{xxx}} \mathrm{a}$ and $\mathrm{VEGF}_{\mathrm{xxx}} \mathrm{b}$ isoforms by fibroblasts may influence the development of IPF.

Methods Normal (NF) and fibrotic fibroblasts (FF) (from patients with proven UIP) were extracted from lung samples using the explant method. The expression of $\mathrm{VEGF}_{\mathrm{xxx}} \mathrm{a}$ and VEGF $_{x x x}$ b by NF and FF was analysed at the mRNA level by RT-PCR and quantified by qPCR. Protein expression was determined by western blotting (WB) and ELISA. We sought to establish a potential functional effect of recombinant $\mathrm{VEGF}_{165} \mathrm{a}$ and $\mathrm{VEGF}_{165} \mathrm{~b}$ proteins on fibroblasts by assessing the expression of a) the extracellular matrix (ECM) protein fibronectin and b) $\alpha$ SMA, a marker of myofibroblast differentiation.

Results Both NF and FF expressed VEGF $_{x x x} a$ and $V_{E G F} F_{x x x} b$ isoforms at the mRNA level as determined by RT-PCR with confirmation by direct sequencing. There was no statistical difference in total VEGF mRNA expression between the two cell types by qPCR ( $\mathrm{p}=0.9307, \mathrm{NF} \mathrm{n}=5, \mathrm{FF} \mathrm{n}=6$ ), but $\mathrm{FF}$ expressed significantly more $V_{E G F} 165$ mRNA than NF $(\mathrm{p}=0.05, \mathrm{NF} \mathrm{n}=5, \mathrm{FF} \mathrm{n}=6)$. Total VEGF protein expression was significantly increased in FF (mean expression 